\title{
ALGEBRAIC $K$-THEORY OF POLY-(FINITE OR CYLIC) GROUPS
}

\author{
BY FRANK QUINN
}

\begin{abstract}
The $K$-theory of the title is described in terms of the $K$ theory of finite subgroups, as generalized sheaf homology of a quotient space. A corollary is that if $G$ is torsion-free, then the Whitehead groups $\mathrm{Wh}_{\imath}(\mathbf{Z} G)$ vanish for all $i$.
\end{abstract}

1. The main result. Suppose that $G$ is a poly-(finite or cyclic) group. Then there is a virtually connected and solvable Lie group $L$ that contains $G$ as a discrete cocompact subgroup. ("Virtually" means a subgroup of finite index has the indicated property.) This follows from results of Auslander and Johnson, as observed in [5]. Let $K$ be a maximal compact subgroup of $L$. Then $G$ acts (on the right) on the contractible manifold $K \backslash L$. The action may not be free; for $y \in(K \backslash L)$ the isotropy subgroup is $G_{y}=\left(y G y^{-1}\right) \cap K$. These isotropy subgroups are finite since they are discrete in the compact group $K$.

Consider the quotient $K \backslash L / G$. Let $[y]$ denote a point with preimage $y$ in $K \backslash L$. Then the isotropy subgroup $G_{y}$ is determined by $[y]$ up to conjugacy. $G_{y}$ therefore defines a "cosheaf up to conjugacy" of groups over $K \backslash L / G$. If $R$ is a ring we can apply the algebraic $K$-theory functor $\mathbf{K}(R[*])$ to this to get a cosheaf of spectra over $K \backslash L / G$. Homology groups with coefficients in a spectral cosheaf are defined $[\mathbf{1 2}]$ and, in this case, denoted by $H_{\imath}\left(K \backslash L / G ; \mathbf{K}\left(R G_{y}\right)\right)$. Homology is discussed further in $\S 2$.

The $K$-theory spectrum used here is the nonconnective one; the lower homotopy groups of the spectrum are Bass's groups $K_{-j}$. Also, maps of $\mathbf{K}(R G)$ induced by conjugation of $G$ are essentially canonically homotopic to the identity. Therefore the uncertainty of definition of the $G_{y}$ vanishes at the spectrum level.

1.1 THEOREM. Suppose $G$ is a discrete cocompact subgroup of a Lie group $L$ that is virtually connected and solvable and has maximal compact subgroup $K$. Suppose $R$ is a subring of the rationals in which the order of the torsion of $G$ is invertible. Then the natural homomorphism

$$
H_{\imath}\left(K \backslash L / G ; \mathbf{K}\left(R G_{y}\right)\right) \rightarrow K_{\imath}(R G)
$$

is an isomorphism for all $i$.

Before discussing the proof we give corollaries and references. First, if $G$ is virtually abelian Yamasaki [18] has proved the analog of 1.1 for the surgery groups of $G$.

Received by the editors October 29, 1984.

1980 Mathematics Subject Classification. Primary 16A54, 18F25, 22E40.

(C) 1985 American Mathematical Society $0273-0979 / 85 \$ 1.00+\$ .25$ per page 
The first corollaries concern Whitehead groups, so we recall their definition. Note that the $K$ groups and the homology groups are both defined as homotopy groups of spectra. The homomorphism of the theorem comes from a map of spectra. The corresponding map with constant coefficients, $\mathbf{H}(B G ; \mathbf{K}(R)) \rightarrow \mathbf{K}(R G)$, coincides with the map defined by Loday [8]. The Whitehead spectrum $\mathrm{Wh}(G ; R)$ is defined to be the cofiber of this map, and the Whitehead groups are the homotopy groups of this spectrum. Formal properties of homology imply

1.2 Corollary. Suppose $G, L, K$ and $R$ are as in 1.1. Then the natural homomorphism

$$
H_{i}\left(K \backslash L / G ; \mathrm{Wh}\left(G_{y} ; R\right)\right) \rightarrow \mathrm{Wh}_{i}(G ; R)
$$

is an isomorphism for all $i$.

If $G$ is torsion free then the isotropy groups $G_{y}$ are all trivial, so the coefficients in the homology group are trivial. This implies

1.3 COROLlaRY. Suppose $G$ is a torsion free poly-(finite or cyclic) group. Then $\mathrm{Wh}_{\imath}(G ; \mathbf{Z})=0$ for all $i$.

Some cases of this were known previously. The cases $i=0,1$ are due to Farrell and Hsiang [5]. They also showed the groups are torsion when $i \geq 0$ [6]. When $G$ is a Bieberbach group (virtually abelian) A. Nicas has shown that $\mathrm{Wh}_{2}(\mathrm{ZG})=0[\mathbf{1 0}], \mathrm{Wh}_{3}$ is 2 -torsion $[\mathbf{9}]$, and $\mathrm{Wh}_{i}$ has no $p$-torsion for $0 \leq i \leq 2 p-2[\mathbf{1 1}]$.

Next we apply a spectral sequence to get more detailed information. Observe that we obtain cosheaves of groups $K_{i}\left(R G_{y}\right)$ over $K \backslash L / G$ by applying $\pi_{i}$ fiberwise to the cosheaves of spectra. There is an Atiyah-Hirzebruch type spectral sequence from ordinary to generalized homology:

$$
H_{\imath}\left(K \backslash L / G ; K_{\jmath}\left(R G_{y}\right)\right) \Rightarrow H_{i+j}\left(K \backslash L / G ; \mathbf{K}\left(R G_{y}\right)\right) .
$$

The leftmost column of this spectral sequence can be described in more familiar terms. Consider the category of (conjugacy classes of) finite subgroups $H \subset G$ with morphisms inclusions of conjugates. Applying $K_{j}$ gives a functor from this category into abelian groups, and we denote by $\lim _{\longrightarrow} K_{j}(R H)$ the direct limit of this functor. The inclusions $H \subset G$ define a homomorphism $\lim _{\longrightarrow} K_{\jmath}(R H) \rightarrow K_{\jmath}(R G)$.

1.4 LemmA. Suppose $L, K, G$ are as in 1.1. Then there is a natural isomorphism $\lim _{\mathrm{l}} K_{j}(R H) \simeq H_{0}\left(K \backslash L / G ; K_{j}\left(R G_{y}\right)\right)$, and the homomorphism to $K_{\jmath}(R G)$ is the inclusion of the $i=0$ column of the spectral sequence.

This is a result of special connectivity properties of strata in $K \backslash L / G$.

The spectral sequence is nearly first quadrant because Carter [2] has shown that $K_{-\imath}\left(R G_{y}\right)=0$ for finite $G_{y}$ and $-i \leq-2$. In particular, the lower left corner gives information about the lowest groups. Using the lemma to replace 
$H_{0}$ terms, we get

1.5 Corollary. Suppose $G, L, K, R$ are as in 1.1. Then

(a) $K_{\imath}(R G)=0$ if $i \leq-2$;

(b) $\lim _{\longrightarrow} K_{-1}(R H) \rightarrow K_{-1}(R G)$ is an isomorphism; and

(c) there is an exact sequence

$$
\begin{aligned}
& \rightarrow H_{2}\left(K \backslash L / G ; K_{-1}\left(R G_{y}\right)\right) \rightarrow \underline{\lim } K_{0}(R H) \rightarrow K_{0}(R G) \\
& \rightarrow H_{1}\left(K \backslash L / G ; K_{-1}\left(R G_{y}\right)\right) \rightarrow 0 .
\end{aligned}
$$

Part (a) has been recklessly conjectured by Hsiang for all $G$, while something like (b) was conjectured by Farrell and Hsiang in [5].

2. Homology. The homology theory used in the theorem is a functor of simplicial maps $p: E \rightarrow X$. We think of $p$ as defining a "coefficient system" over $X$. Suppose $\mathbf{J}$ is a covariant (and "continuous") functor from spaces to spectra. $\mathbf{J}$ can be applied to the point inverses of $p$ to yield a "spectral sheaf" over $X$, denoted $\mathbf{J}(p)$. Homology groups $H_{i}(X ; \mathbf{J}(p))$ are defined in the appendix to $[\mathbf{1 2}]$ and in more detail in Chapter 2 of [14].

Now suppose a discrete group $G$ acts simplicially on a complex $X$. Let $E G$ denote a contractible complex on which $G$ acts freely, and let $p$ denote the projection in the balanced product construction $p:(E G \times X) / G \rightarrow X / G$. Applying homology gives groups $H_{i}(X / G ; \mathbf{J}(p))$. The map fits into a diagram

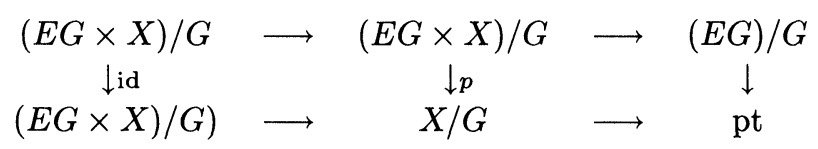

The right vertical map has range a point, so the homology groups are just the coefficient groups $\pi_{\imath} \mathbf{J}(B G)$. The left vertical gives constant coefficient homology $H_{i}((E G \times X) / G ; \mathbf{J}(\mathrm{pt}))$. The diagram defines natural homomorphisms

$$
H_{\imath}((E G \times X) / G ; \mathbf{J}(\mathrm{pt})) \rightarrow H_{i}(X / G ; \mathbf{J}(p)) \rightarrow \pi_{i} \mathbf{J}(B G) .
$$

If $X$ is contractible, $(E G \times X) / G \simeq B G$. Assuming this and letting $\mathbf{J}=$ $\mathbf{K}\left(R \pi_{1}\right)$, we get homomorphisms

$$
H_{i}(B G ; \mathbf{K}(R)) \rightarrow H_{i}\left(X / G ; \mathbf{K}\left(R \pi_{i}(p)\right)\right) \rightarrow K_{\imath}(R G) .
$$

The center group is the one used in $\S 1$, where the notation $\mathbf{K}\left(R G_{y}\right)$ is used for the coefficient system. This notation reflects the fact that the point inverse $p^{-1}(y)$ is homotopy equivalent to $B G_{y}$. The right homomorphism is the natural homomorphism referred to in the main theorem, and the composition of the two is the Loday homomorphism.

3. Sketch of the proof. The proof follows the general outline used by Farrell and Hsiang [5] in the $i=0,1$ cases. The major new ingredient is a controlled version of algebraic $K$-theory [14].

Suppose $E$ is a space. There is a category with morphisms formal sums (with $R$ coefficients) of paths in $E[\mathbf{1 3}]$. "Matrices" of these can be defined and used to construct an analog of the $K$-theory of Volodin [15]. If $p: E \rightarrow X$ 
is a map to a metric space, we can additionally impose restrictions on the diameter of the images of the paths in $X$. This gives an " $\varepsilon$-controlled" $K$ theory. Finally the inverse limit of this as $\varepsilon \rightarrow 0$ gives the fully controlled theory. The resulting controlled $K$ groups are denoted $K_{\imath}(X ; p, R)$.

A basic result of the subject is that the controlled groups are isomorphic to homology groups. Specifically, suppose $X$ is a finite complex and $p$ is simplicial. Then the controlled group is isomorphic to $H_{i}\left(X ; \mathbf{K}\left(R \pi_{1} p^{-1}(y)\right)\right)$. When $p$ is the balanced product projection, as in $\S 2$, this is exactly the homology used in Theorem 1.1. The conclusion is that to show that something in $K_{i}(R G)$ comes from homology, it is sufficient to show that it can be represented by a controlled object with small diameter.

Now suppose $L$ is a Lie group containing $G$, as in 1.1. Let $L^{\prime}$ denote the commutator of the identity component of $L .\left(L^{\prime} K\right) \backslash L$ is isometric to euclidean space, of positive dimension since $L$ is virtually solvable. $G$ acts on this by isometries, so the image of $G$ in the automorphism group of $\left(L^{\prime} K\right) \backslash L$ is a cyrstallographic group (see eg. Farkas [4]). It is sufficient to control sizes in $\left(L^{\prime} K\right) \backslash L$, because the other directions in $K \backslash L$ are parametrized by $L^{\prime}$, which has smaller dimension than $L$. We may assume that control can be obtained in groups of smaller dimension than $L$ as an induction hypothesis.

The reason crystallographic actions are useful is that there are a lot of expansive maps. If $\Gamma$ and $\Delta$ are crystallographic groups acting on $\mathbb{R}^{n}$, then an $s$-expansive map is a diffeomorphism $\mathbb{R}^{n} \rightarrow \mathbb{R}^{n}$ that is equivariant with respect to a homomorphism $\tau: \Gamma \rightarrow \Delta$ and increases distances by a factor of $s$. Pulling back through this map reduces distances by $s$, and so gives geometric control. Pulling back corresponds to the algebraic operation of restriction res: $K_{\imath}(R \Delta) \rightarrow K_{i}(R \Gamma)$. A corollary is that if $h \in K_{\imath}(R \Delta)$ then there is $s$ so that if $\tau$ corresponds to an $s$-expansive map then ind(res $(h))$ lies in the image of the homology group. Here ind: $K_{i}(R \Gamma) \rightarrow K_{i}(R \Delta)$ is the induction map.

The hyperelementary induction theorem in this context states that if $\theta: \Delta$ $\rightarrow \pi$ is a homomorphism to a finite group, then elements of $K_{i}(R \Delta)$ can be written as sums of elements of the form ind(res $(h))$, inductions of restrictions to subgroups of the form $\theta^{-1}(H)$, where $H$ is hyperelementary in $\pi$. $\Delta$ itself has a free abelian subgroup of finite index. The strategy is now this: assume the theorem for crystallographic groups with free abelian groups of smaller index. Then contrive finite quotients $\Delta \rightarrow \pi$ so that inverses of hyperelementary subgroups of $\pi$ either have smaller index or correspond to expansive maps. In either case ind $(\operatorname{res}(h))$ is an element of the homology, so the induction theorem implies that all elements of $K_{i}(R \Delta)$ come from homology.

If $\Delta$ has a crystallographic quotient with no 1-dimensional images, then we are in case (ii) of Theorem 1.1 of [5]. In this case the strategy outlined above is successful. If there is a nonabelian 2-dimensional image which has a 1-dimensional image, we are in a situation like that of [6], with a few new cases. In this case the strategy can be modified to get control in a 1-dimensional image. Finally, if neither of the above occur, then we are reduced to considering $\mathrm{Z} \times \pi$ or an extension $\mathrm{Z} \rightarrow G \rightarrow \pi$, with $\pi$ finite. These cases can be expressed as generalized free products to which the theorem of Waldhausen [16] applies. 
It is at this point that the hypothesis on $R$ is necessary; it ensures that the amalgamating rings are regular, so the Nil terms in Waldhausen's theorem vanish.

4. Remarks. Suppose that $G$ acts on $X$ and $R$ is a ring. There is a map of spectra $\mathbf{H}\left(X / G ; \mathbf{K}\left(R G_{y}\right)\right) \rightarrow \mathbf{K}(R G)$, the spectrum-level version of the homomorphisms described in $\S 2$. Denote the cofiber (in the category of spectra) of this map by $\operatorname{Nil}(G, X ; R)$ and its homotopy groups, by $\operatorname{Nil}_{i}(G, X ; R)$. These fit in a long exact sequence with the homology and $K$ groups, so measure the extent to which the main theorem fails.

If $G$ acts freely on $X$ and $X$ is contractible, then the definition coincides with the definition of the Whitehead groups: $\mathrm{Wh}_{\imath}(G, R)=\operatorname{Nil}_{i}(G, E G ; R)$. The classical Nil summand of $K_{0}(R(\mathbf{Z} \times G))$ fits in as follows: $\mathbf{Z} \times G$ acts on the reals $\mathbb{R}$ by the usual action of $\mathbf{Z}$, and $\operatorname{Nil}(G, R)=\operatorname{Nil}_{0}(G \times \mathbf{Z}, \mathbb{R} ; R)$. Generalized free product structures on groups correspond to actions of the groups on trees, so Waldhausen's Nil groups [16] also can be seen this way. Waldhausen's results give the following.

(Waldhausen) Suppose $X$ is a tree, on which $G$ acts cellularly. Then and

(a) the exact sequence splits; $K_{i}(R G) \simeq H_{i}\left(X / G ; \mathbf{K}\left(R G_{y}\right)\right) \oplus \operatorname{Nil}_{i}(G, X ; R)$;

(b) $\operatorname{Nil}_{\imath}(G, X ; R)$ vanishes if $R$ is a subring of the rationals, and the isotropy subgroups are finite with orders invertible in $R$.

The order condition on the isotropy subgroups ensures that the amalgamating rings are regular. We note the remarkable characterization of Dunwoody (see Dicks [3]) that a group $G$ admits an action on a graph satisfying (b) if and only if the augmentation ideal in $R G$ is projective.

Questions. Suppose $G, L$, and $K$ are as in 1.1 , but $R$ is arbitrary.

(a) Does the exact sequence split as in the tree case, so

$$
K_{i}(G R) \simeq H_{\imath}\left(K \backslash L / G ; \mathbf{K}\left(R G_{y}\right)\right) \oplus \operatorname{Nil}_{\imath}(G, K \backslash L ; R) ?
$$

(b) Is $\mathrm{Nil}_{i}(G, K \backslash L ; R)$ a torsion group-for example, with exponents dividing powers of exponents of the torsion of $G$ that are not invertible in $R$ ?

We note that the proof of 1.1 would yield (b) if it were known for appropriate generalized free products (cases where $X=\mathbb{R}$ ). Some of these cases follow from results of Weibel $[\mathbf{1 7}, 6.4]$. The question is supported by calculations for abelian $G[\mathbf{1}]$.

Finally, one might hope for similar results for discrete subgroups of other Lie groups. Unfortunately, rigidity theorems show that there are usually rather few expansive maps, so the proof used here cannot work.

\section{REFERENCES}

1. H. Bass and M. P. Murthy, Grothendieck groups and Picard groups of abelian group rings, Ann. of Math. 86 (1967), 16-73.

2. D. W. Carter, Lower K-theory of finite groups, Comm. in Algebra 8 (1980), 1927-1937.

3. W. Dicks, Groups, trees, and projective modules, Lecture Notes in Math., vol. 780, SpringerVerlag, 1980. 
4. D. R. Farkas, Crystallographic groups and their mathematics, Rocky Mountain J. Math. 11 (1981), 511-551.

5. F. T. Farrell and W. C. Hsiang, The Whitehead group of poly-(finite or cyclic) groups, J. London Math. Soc. 14 (1981), 308-324.

6. - On the rational homotopy groups of the diffeomorphism groups of discs, spheres, and aspherical manifolds, Proc. Sympos. Pure Math., vol. 32, Amer. Math. Soc., Providence, R.I., 1978, pp. 3-22.

7. (to appear).

8. J. L. Loday, K-theorie algebraique et representations des groupes, Ann. Sci. École Norm. Sup. 9 (1976), 309-377.

9. A. J. Nicas, On $\mathrm{Wh}_{2}$ of a Bieberbach group, Topology (to appear).

10. __ On $\mathrm{Wh}_{3}$ of a Bieberbach group, Proc. Cambridge Philos. Soc. 95 (1984), 55-60.

11. __ On the higher Whitehead groups of a Bieberbach group, Trans. Amer. Math. Soc. (to appear).

12. F. Quinn, Ends of maps. II, Invent. Math. 68 (1982), 353-424.

13. ___ Geometric algebra, Proc. 1983 Rutgers Topology Conf. Springer Lecture notes (to appear).

14. __ Controlled algebra and topology, Lecture Notes (in preparation).

15. I. A. Volodin, Algebraic K-theory as extraordinary homology theory on the category of assocrative rings with unity, Math. USSR Izv. 5 (1971), 859-887.

16. F. Waldhausen, Algebraic K-theory of generalized free products, Ann. of Math. (2) 108 (1978), 135-256.

17. C. A. Weibel, Meyer Vietoris sequences and module structures on $N K_{*}$, Algebraic $K$ Theory (Evanston, 1980), Lecture Notes in Math., vol, 854, Springer-Verlag, 1981.

18. M. Yamasaki, Surgery groups of crystallographic groups, Dissertation, Virginia Tech., 1982.

Department of Mathematics, Virginia Polytechnic Institute and StATE UNIVERSity, BLACKSBURG, Virginia 24061 